\title{
Heterotaxy Polysplenia Syndrome in an Old Female
}

\author{
Mohamed Hajri1, 3*, Maroua Yangui2, 3, Ghofran Talbi 1, 3, Wael Ferjaoui*1, 3, Leila Ben Farhat 2, 3, Rached Bayar ${ }^{1,3}$
}

\begin{abstract}
(c) (1) (2) (2)
DOI: https://doi.org/I0.3329/jom.v23il.5794I

Copyright: (C) 2022 Hajri M. This is an open access article published under the Creative Commons Attribution-NonCommercial-NoDerivatives 4.0 International License, which permits use, distribution and reproduction in any medium, provided the original work is properly cited, is not changed in any way and it is not used for commercial purposes.
\end{abstract}

Received: 15 November, 202I;

Accepted: 25 December, 2021

A 74-year-old woman, with no medical history, presented to the emergency room with epigastric pain for three days. On physical examination, vital signs were all within normal limit, and abdominal palpation revealed epigastric tenderness. Chest and abdominal CT scan was performed and showed typical findings of COVID-19 pneumonia, affecting $20 \%$ of lung parenchyma (Fig. 1). CT scan also showed multiple splenic nodules (about eight) in different sizes ranging from

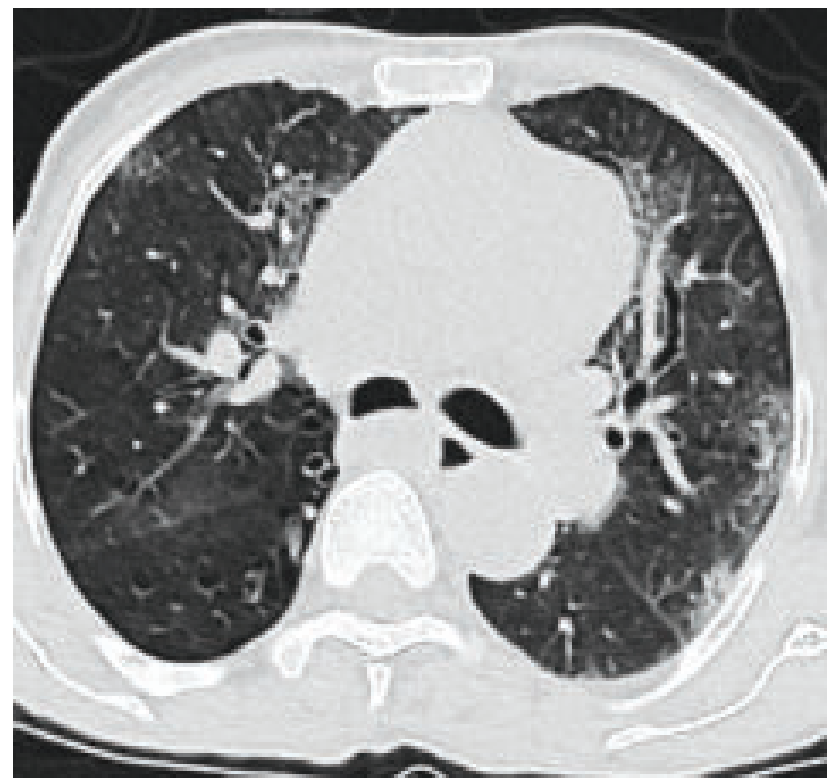

Fig. 1: Chest computed tomography showing COVID-19 pneumonia, affecting $20 \%$ of lung parenchyma

1. Department of General surgery, Mongi Slim University Hospital, Tunis, Tunisia

2. Department of Radiology, Mongi Slim University Hospital, Tunis, Tunisia

3. Faculty of Medicine of Tunis, Tunis El Manar University, Tunis, Tunisia

*Corresponding author: Mohamed Hajri, Department of General surgery, Mongi Slim University Hospital, Tunis, Tunisia. Tel: +216 52430099/E mail: farjaouiwael4@gmail.com https://orcid.org/00000002-4279-9370
3 to $6,5 \mathrm{~cm}$ (Fig. 2), the superior mesenteric artery (SMA) was to the right of the superior mesenteric vena (SMV) (Fig. 3 ), the small bowel was right-sided and the colon was leftsided, dilated azygos vein with agenesis of the inferior vena cava (IVC) and agenesis of the dorsal pancreas (Fig. 3). The patient was hospitalized in a Covid-19 unit. She was discharged after six days of oxygen therapy and medical treatment.

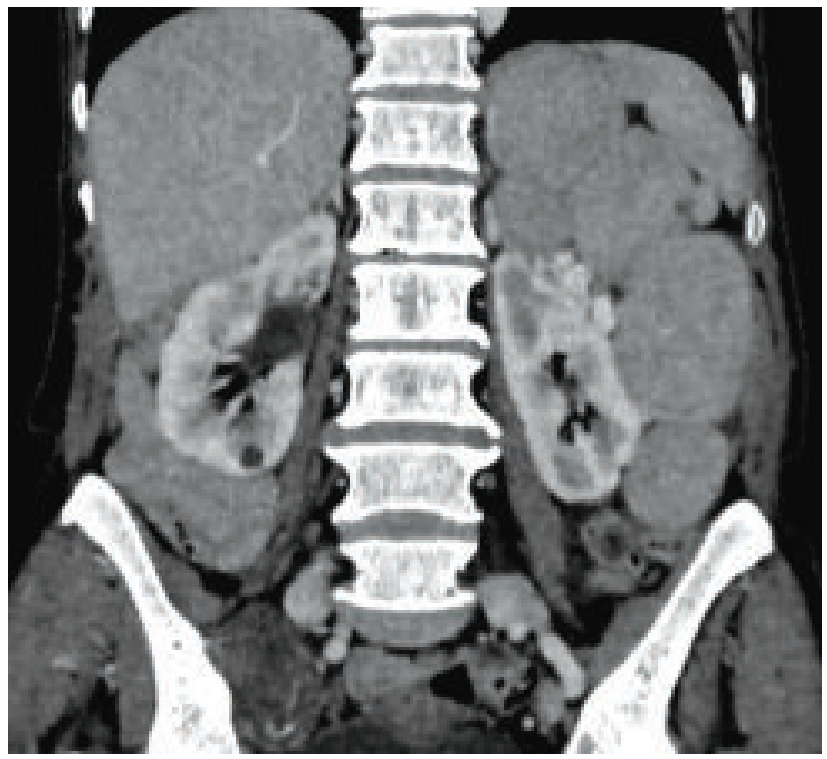

Fig. 2: Abdominal computed tomography showing presence of multiple spleens in different sizes

Heterotaxy syndrome (HS) (or situs ambiguus) is a rare condition occurring in approximately $1 / 250,000$ births, in which there is an abnormal arrangement of thoracoabdominal organs. ${ }^{1}$ It's the result of an early embryological developmental failure. ${ }^{2}$ Polysplenia syndrome (PS) (or left isomerism) is the subtype of HS with features of bilateral left-sidedness. As in our case, typical findings are: presence of multiple spleens, truncated pancreas with agenesis of the dorsal pancreas, interrupted IVC with azygous continuation and intestinal non rotation with common mesentery. It can 


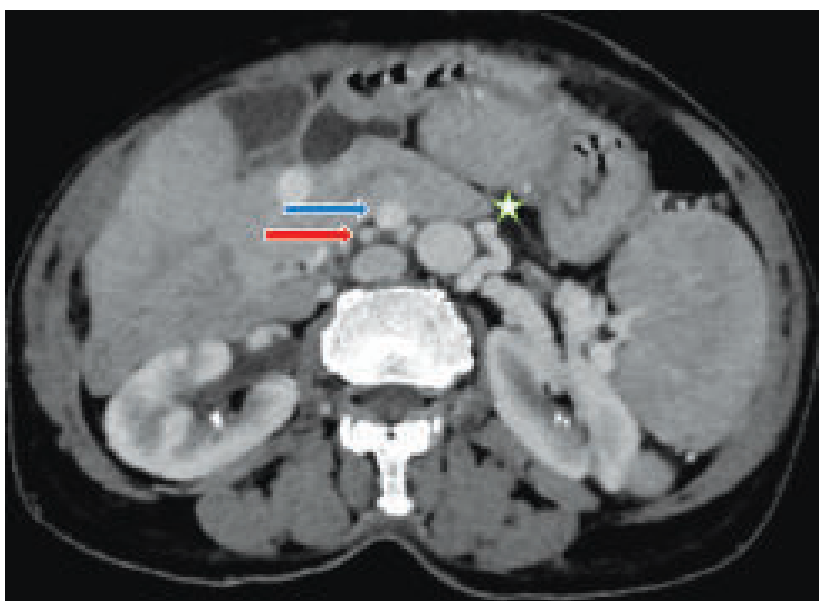

Fig. 3: Abdominal computed tomography showing agenesis of the dorsal pancreas (Asterisk) with inversion of superior mesenteric vessels (SMA (red arrow) to the right of the VMA (blue arrow)) also include a midline liver with or without biliary abnormality and a midline or right-sided aorta. ${ }^{3}$

\section{References:}

1. Low J, Williams D, Chaganti J. Polysplenia syndrome with agenesis of the dorsal pancreas and preduodenal portal vein presenting with obstructive jaundice-a case report and literature review. Br J Radiol. The British Institute of Radiology. 36 Portland Place, London, W1B 1AT; 2011;84(1007):e219 22.

2. Fulcher AS, Turner MA. Abdominal manifestations of situs anomalies in adults. Radiographics. Radiological Society of North America; 2002;22(6):1439 56.

3. Renier H. Intra-Abdominal Abnormalities Associated with Polysplenia Syndrome. J Belg Soc Radiol. Ubiquity Press; 2019;103(1). 